\title{
Les sépultures individuelles campaniformes en
}

\section{France}

Jean-Yves Noël

\section{OpenEdition}

1 Journals

Édition électronique

URL : http://journals.openedition.org/rao/2216

DOI : 10.4000/rao.2216

ISBN : 978-2-7535-3432-2

ISSN : 1775-3732

\section{Éditeur}

Presses universitaires de Rennes

\section{Édition imprimée}

Date de publication : 25 décembre 2013

Pagination : 309-310

ISBN : 978-2-7535-3430-8

ISSN : 0767-709X

\section{Référence électronique}

Jean-Yves Noël, «Les sépultures individuelles campaniformes en France », Revue archéologique de l'Ouest [En ligne], 30 | 2013, mis en ligne le 25 décembre 2013, consulté le 06 décembre 2020. URL : http://journals.openedition.org/rao/2216; DOI : https://doi.org/10.4000/rao.2216

Ce document a été généré automatiquement le 6 décembre 2020.

Tous droits réservés 


\title{
Les sépultures individuelles campaniformes en France
}

\author{
Jean-Yves Noël
}

\section{RÉFÉRENCE}

Salanova L. et Tcheremissinoff Y. (dir.), «Les sépultures individuelles campaniformes en France », Gallia Préhistoire, XLI ${ }^{\mathrm{e}}$ Supplément, 2011 (ISBN 978-2-271-07124-8, 49€)

1 Les sépultures individuelles de la fin du III ${ }^{\mathrm{e}}$ millénaire avant notre ère sont encore rares sur le territoire national. Cependant, cette faiblesse quantitative est aujourd'hui compensée par la qualité de l'information qu'elles fournissent. En effet, par leur mode d'approche (étude du contenant et du contenu, datations isotopiques...), les découvertes récentes apparaissent aujourd'hui comme les données les plus fiables pour aborder les différentes influences qui traversent la France à cette époque et préparent les futures sociétés de l'âge du Bronze. Un premier tableau de ce paysage funéraire particulier, majoritairement propre à la civilisation campaniforme, peut donc être brossé. Pour l'aborder, les directrices de cette publication ont choisi de faire appel à des chercheurs de différents horizons (géographiques et professionnels) afin qu'ils présentent leurs données sous forme de courtes monographies ou de petites synthèses régionales comme celle effectuée par C. Billard pour la Normandie (Chap. III) et celle de A. Lefebvre et al. pour la Lorraine (Chap. VIII). Ces contributions, nommées " chapitres ", sont rassemblées et présentées en deux grandes entités, le Nord et le Sud de la France.

2 Le Nord livre le plus grand nombre de sépultures individuelles attribuées à la culture campaniforme. Les chapitres offrent un large panel de sites bien conservés, de la Vienne (sépulture de la Folie à Poitiers, Tchéremissinoff et al., Chap. I) au Luxembourg (sépultures d'Altwies, Le Brun-Ricalens et al., Chap. IX), issus pour l'essentiel de l'archéologie préventive de ces dix ou vingt dernières années. Le réexamen des données de la sépulture d'Arenberg dans le Nord, découverte en 1969, fait alors figure 
d'exception (Salanova et al., Chap. VII). Les monographies de ces sites sont très complètes. Les données sont exposées clairement selon une architecture récurrente. Elle débute par le contexte de découverte puis la description de la structure pour ensuite aborder le défunt, son milieu de décomposition et son mobilier d'accompagnement. Chaque élément de ce dernier fait alors l'objet d'un examen approfondi, à l'exemple des études pétrographiques proposées par F. Convertini pour de nombreux vases ou encore celles sur la composition élémentaire du mobilier métallique. Quand elles sont disponibles, les datations absolues réalisées sur les os du défunt sont systématiquement exposées. La synthèse proposée pour cette partie de la France, par Laure Salanova, dans le chapitre X, débute d'ailleurs par ces aspects chronologiques. Elle propose une modélisation en trois étapes s'échelonnant de 2500 à 1950 av. J.-C. Des comparaisons deviennent alors possibles avec les modèles voisins : britannique, hollandais et centro-européens. Elle met ensuite en évidence des pratiques récurrentes, comme l'emploi de fosses architecturées complexes (Ciry-Salsogne dans l'Aisne, Hachem et al., Chap. II ; Pouilly-en-Moselle ou encore Hatrize en Meurthe-etMoselle), ou le dépôt d'assemblages types par période. Enfin, elle note différentes influences dans les facteurs d'évolution de ces sépultures individuelles entre la fin du Néolithique et le début de l'âge du Bronze.

3 Le Sud de la France est moins documenté. À l'image de la Bretagne, il semblerait que cela soit imputable à une plus grande place donnée à l'emploi/réemploi des sépultures collectives dans les modes d'inhumation de cette époque. Toujours est-il que les exemples proposés dans les chapitres XI à XIII sont particulièrement pertinents et originaux. Il s'agit de fosses aménagées avec des empierrements comme la sépulture de La Fare à Forcalquier (Lemercier et al.), de coffres comme celui du site Georges Besse II-5 à Nîmes (Tchérémissinoff et al.), ou encore d'une sépulture d'enfant sous grotte à Montagnac-Montpezat (Courtin et al.). Ces pratiques complètent la variabilité de celles du Nord de la France. Elles documentent aussi des aspects symboliques moins perceptibles dans les tombes en matériaux périssables de la sphère septentrionale. Les chapitres concernant la «Grotte murée » à Montagnac et le coffre de «Georges Besse II-5 » à Nîmes sont particulièrement édifiants à ce sujet. Le déficit d'exemples concrets et illustrés est compensé par la synthèse proposée par O. Lemercier et Y. Tchérémissinoff dans le chapitre XIV. Ce dernier dresse un portrait évolutif des pratiques funéraires, du Néolithique final au Bronze ancien, du Sud-Ouest atlantique au Jura en passant par le Massif central, la côte méditerranéenne et le couloir du Rhône. Cependant, la richesse des informations qui le compose, présentées selon un plan à la fois chronologique, géographique et thématique, le rend parfois difficile d'accès.

4 A contrario, la conclusion générale du chapitre $\mathrm{XV}$, proposée par Y. Tchérémissinoff et L. Salanova, résume ces pratiques à l'échelle de la France, en quelques pages. Elle reprend, par de courts paragraphes, la grille de lecture proposée en introduction: le contexte des tombes, leur architecture, le ou les défunts et, enfin, le mobilier funéraire. Les auteurs terminent alors sur la question du lien, voire de la filiation, qui peut être établi entre ces pratiques campaniformes et celles de l'âge du Bronze ancien pour lesquelles une individualisation et une certaine mise en valeur du défunt sont de mise. La symbolique, induite par cette évolution, reste difficile à percevoir. Néanmoins, elle reflète des transformations sociales évidentes par rapport au Néolithique final.

5 Pour finir, l'organisation, la qualité des contributions et des illustrations font de cet ouvrage un véritable manuel. Si les synthèses peuvent rapidement contenter le 
chercheur ou l'étudiant, les présentations de sites raviront simplement tous les curieux.

\section{AUTEURS}

JEAN-YVES NOËL

Conseil Général d'Eure-et-Loir 